\title{
BMJ Pregnancy outcomes following maternal Open exposure to second-generation antipsychotics given with other psychotropic drugs: a cohort study
}

\author{
Alexander Sadowski, ${ }^{1}$ Michelle Todorow, ${ }^{1,2}$ Parvaneh Yazdani Brojeni, ${ }^{1,3}$ \\ Gideon Koren, ${ }^{1,3}$ Irena Nulman ${ }^{1,3}$
}

To cite: Sadowski A, Todorow M, Yazdani Brojeni $\mathrm{P}$, et al. Pregnancy outcomes following maternal exposure to secondgeneration antipsychotics given with other psychotropic drugs: a cohort study. BMJ Open 2013;3:e003062. doi:10.1136/bmjopen-2013003062

- Prepublication history for this paper is available online. To view these files please visit the journal online (http://dx.doi.org/10.1136/ bmjopen-2013-003062).

Received 18 April 2013 Revised 10 June 2013 Accepted 14 June 2013

This final article is available for use under the terms of the Creative Commons Attribution Non-Commercial 3.0 Licence; see http://bmjopen.bmj.com

For numbered affiliations see end of article.

Correspondence to Dr Irena Nulman; irena. nulman@sickkids.ca

\section{ABSTRACT}

Objectives: Second-generation antipsychotics (SGAs), in conjunction with other psychotropic medications, are increasingly used to treat psychiatric disorders in pregnancy. The few available studies investigating the reproductive safety of SGAs did not reach conclusive results, and none have compared monotherapy with polytherapy involving other psychotropic medications.

Design: Descriptive cohort study using a prospectively collected database.

Setting: Motherisk Program, The Hospital for Sick Children, Toronto, Canada.

Participants: 133 women exposed to SGAs and other psychotropic drugs and 133 matched healthy controls were assessed and analysed. Outcomes of mother-child pairs exposed to SGAs in monotherapy ( $\mathrm{N}=37$ ) were compared with those exposed to SGAs with other psychotropic medications (in polytherapy; $\mathrm{N}=96$ ).

Main outcome measures: Maternal, pregnancy, delivery and neonatal outcomes.

Results: $72 \%$ of exposed women received SGAs in polytherapy, and 101 women took their medications throughout pregnancy. These women had significantly higher pre-pregnancy weight, experienced more associated comorbidities and instrumental deliveries, and delivered a greater proportion of large for gestational age neonates. There were no differences in maternal weight gain in pregnancy between the exposed and comparison groups and between the monotherapy-exposed and polytherapy-exposed subgroups. The exposed neonates were more likely to be born premature, were admitted more often to the neonatal intensive care unit, presented with poor neonatal adaptation signs and had higher rates of congenital malformations. All the aforementioned neonatal outcomes were found mainly in the polytherapy subgroup.

Conclusions: The use of SGAs in polytherapy was prevalent in the assessed cohort and was associated with adverse pregnancy outcomes for both the mother and the child. In utero exposure to SGA monotherapy appears to be associated with less risk to the fetus. Future research should focus on polytherapy in pregnancy in order to define its reproductive safety and to separate the effects of medication exposure, underlying psychopathology and associated comorbidities.

\section{ARTICLE SUMMARY}

Article focuses

- To investigate the reproductive safety of the second-generation (atypical) antipsychotic (SGA) medications.

- To compare pregnancy outcomes following maternal use of SGA monotherapy and polytherapy involving other psychotropic medications.

Key messages

- The use of SGAs in polytherapy in pregnant women with mental health disorders is presently a common practice and is associated with adverse pregnancy outcomes for both the mother and the child.

- In utero exposure to SGA monotherapy appears to be associated with less risk to the fetus.

- The reproductive safety of antipsychotic medications should be studied in the reality of polytherapy, associated comorbidities and environmental and genetic confounders.

\section{INTRODUCTION}

Psychiatric disorders are among the most common pathologies affecting women of childbearing age. Recent estimates of the prevalence of psychiatric disorders in pregnancy range from $14 \%$ to $30.5 \%^{1-3}$ and include mood, anxiety and psychotic disorders. ${ }^{1-4}$ Untreated mental illness during pregnancy is associated with risks to both the mother and the child. ${ }^{5}$ Uncontrolled schizophrenia, depression and bipolar disorder have all been associated with an increased risk for adverse pregnancy outcomes. ${ }^{6-8}$ The risks associated with uncontrolled mental illness often lead physicians to recommend maintenance of treatment with psychotropic medications throughout gestation.

Antipsychotics have been utilised in the treatment of psychiatric disorders for a number of decades. While the initial drugs, now referred to as first-generation 


\section{ARTICLE SUMMARY}

Strengths and limitations of this study

- A cohort study using a prospectively collected database, including comparison groups and matching.

- At present, the largest study to investigate pregnancy outcomes following the maternal use of SGA in monotherapy and in polytherapy, identifying risk behaviours and factors associated with adverse pregnancy outcomes.

- Inability to address confounding by indication and separate the effects of maternal mental illness and its severity from psychotropic pharmacotherapy on pregnancy outcome.

- The women who contacted Motherisk tended to be middle to upper middle class, whereas a smaller proportion of callers came from the lower socioeconomic and/or immigrant population; however, this may not affect the generalisability of the results, as their outcomes may be confounded by other factors unrelated to the drug effect.

- Although the women were recruited prospectively, there are potential limitations in the retrospectivity of a database.

- The sample size necessary to define a twofold increase of malformation rates of $1-3 \%$ above the baseline was insufficient.

antipsychotics (FGAs), demonstrated a profound effect on treatment outcomes, this group of medications is not only associated with serious extrapyramidal complications, but is also known to induce hyperprolactinaemia and to affect fertility. The existing literature on pregnancy outcomes following exposure to FGAs has not shown a significantly increased risk for malformations above the baseline incidence. Conversely, many FGAs (including haloperidol, chlorpromazine, fluphenazine, thioridazine) have been associated with perinatal complications, particularly when exposure is later in pregnancy. ${ }^{9}$ These include but are not limited to withdrawal symptoms, unstable body temperature, extrapyramidal signs, respiratory distress, seizures and transient neurodevelopmental delay. Conclusions regarding other FGAs are limited due to either poor methodology or incomplete reporting. ${ }^{9}$ Although the reproductive safety of FGAs is as yet unconfirmed, the adverse side effect profile necessitated the development of newer medications in recent years. Second-generation antipsychotics (SGAs), also known as atypical antipsychotics, are a group of medications primarily used in the treatment of schizophrenia and bipolar disorder. Since the mid-1990s, the use of SGAs has increased drastically and expanded rapidly to the treatment of depressive and anxiety disorders. ${ }^{10-13}$ Furthermore, deinstitutionalisation, the destigmatisation of mental illness and the improved side effect profile of SGAs, particularly the reduced incidence of hyperprolactinaemia in most SGAs, have all contributed to increased fertility rates among treated women. ${ }^{9}$ The rise in birth rate in women with psychiatric disorders, combined with the increase in off-label usage of these medications, has resulted in a significant increase in the number of fetuses exposed to this newer class of antipsychotics. While SGAs readily cross the placenta, ${ }^{14}$ the existing studies that have investigated the reproductive safety of these agents did not reach conclusive results due to the methodological limitations, small sample sizes and failure to assess SGAs in monotherapy.

The meagre body of literature regarding pregnancy outcomes following the maternal use of SGAs has produced conflicting findings, which may be attributed to the different methodologies and inadequate control for confounding variables. ${ }^{15}$ A number of studies have found that exposure to SGAs is associated with a significantly increased risk of low birth weight ${ }^{16-18}$ and small for gestational age infants, in comparison to healthy controls. ${ }^{17}$ Conversely, Lin $e t a l^{17}$ found that the risk of low birth weight, preterm births and small/large for gestational age (LGA) infants did not differ between women with schizophrenia receiving SGAs and those with the disorder who did not use an antipsychotic drug. In contrast, Newham et al ${ }^{19}$ found that neonates exposed to SGAs $(n=25)$ were significantly more likely to be LGA compared with healthy controls. Furthermore, a large study utilising data from the Swedish Medical Birth Register reported an increased risk of congenital malformations associated with exposure to either a typical antipsychotic or SGA; however, this finding failed to reach statistical significance after controlling for exposure to anticonvulsants. ${ }^{18}$

Another growing concern is the increased use of multiple psychotropic medications in the treatment of mental illness. A number of studies have shown a significant rise in the incidence of polytherapy with two or more antidepressants, antipsychotics and sedative-hypnotics, as well as an increase in the concomitant prescription of an antidepressant and antipsychotic. ${ }^{15} 16$ 19-24 Although polytherapy involving SGAs and other psychotropic medications appears to be increasingly common, no study until now has directly compared the reproductive safety of SGA monotherapy versus polytherapy with other medications. We aimed to investigate the pregnancy outcomes of women receiving SGAs relative to a comparison group of matched healthy controls and to compare the outcomes of antipsychotic monotherapy with polytherapy involving other psychotropic medications.

\section{METHODS AND MATERIALS}

A descriptive cohort study with matched controls using a prospectively collected database was conducted.

All potential participants were identified from a database of women who directly contacted the Motherisk Program at the Hospital for Sick Children in Toronto, Canada, between 2005 and 2009. Motherisk is a free counselling service offering evidence-based information regarding the reproductive safety of medications and other potentially teratogenic agents to pregnant and breastfeeding women across Canada and the USA. Women who initially called the service to inquire about the safety of an SGA and who confirmed the use of this medication for a minimum of 4 weeks of pregnancy were invited to participate. Women who were exposed to 
teratogenic medications unrelated to their psychiatric disorder treatment, such as acutane, or who abused substances (eg, alcohol, marijuana, cocaine, heroin, etc) were excluded from the study cohort.

A comparison group was comprised of women who contacted Motherisk between 2005 and 2009, and reported exposure to non-teratogenic agents (eg, acetaminophen, antihistamines, etc). Women in the exposed and comparison groups were matched for age at conception ( \pm 3 years) and pregnancy duration at the initial time of contact $( \pm 2$ weeks). Fertility-assisted pregnancies, twin/triplet pregnancies or pregnancies with known outcomes at the initial time of contact (eg, contacted Motherisk following the birth, reported abnormal pregnancy screening tests and/or ultrasounds) were excluded from the exposed and comparison groups. Moreover, control women who reported a history of psychiatric disorders or who were exposed in their current pregnancy to a known teratogen were excluded.

Women in the exposed and comparison groups participated in follow-up telephone interviews between 2009 and 2012, which gathered information regarding pregnancy, delivery and neonatal outcomes in order to define the variables of interest. Maternal medical and obstetric histories and type and duration of SGAs and other psychotropic medications used in pregnancy were documented. Information on usage of over-the-counter and prescription medications, substance use and breastfeeding was collected as well. Consent was obtained to request a report from the child's physician, which included hospital birth records, postnatal assessments and information on congenital malformations and the child's health. Data obtained from physicians were crossreferenced with information provided by the mothers in order to increase accuracy and minimise recall bias. Oral consent for participation was provided by all women over the phone.

All study procedures were approved by The Hospital for Sick Children Research Ethics Board.

\section{Statistical analysis}

The first statistical comparison was performed between those exposed to SGAs and matched unexposed healthy controls. The second statistical comparison was between those receiving SGA monotherapy and those receiving SGAs along with other psychotropic medications (defined by us as being exposed to polytherapy). Maternal characteristics, as well as pregnancy, delivery and neonatal outcomes, were compared between groups. The Shapiro-Wilk and Levene tests were used to assess normality and homogeneity of variance of data, respectively. Continuous data were compared between groups using independent $t$ tests and Mann-Whitney $\mathrm{U}$ tests when data violated assumptions. All tests were twotailed. All categorical outcomes were analysed using Pearson's $\chi^{2}$ or Fisher's exact tests. Multiple linear and logistic regression models were constructed to investigate significant predictors of the outcomes of interest.

\section{RESULTS}

From 2005 to 2009, 370 women called Motherisk regarding SGA counselling. Among them, 107 women could not be reached at their last reported contact details, 20 refused participation and 110 did not meet the inclusion criteria. The intake forms of women who were lost to follow-up or declined to participate were analysed. Their medical and psychiatric histories, medications, concomitant disorders and demographic characteristics did not differ from those of the included cohort.

Therefore, the current study includes 133 women who received SGA medications during pregnancy and 133 matched healthy women $(\mathrm{N}=266)$. The majority of women in the exposed group reported taking quetiapine $(69.9 \%)$, followed by olanzapine $(16.5 \%)$, risperidone $(10.5 \%)$, aripiprazole $(1.5 \%)$, paliperidone $(0.8 \%)$ and quetiapine plus olanzapine $(0.8 \%)$. The most common indications for psychotropic medication use were bipolar disorder (36.8\%), depression (27.1\%), anxiety and depression (9.8\%) and sleep disorders $(9.8 \%)$. Schizophrenia and schizoaffective disorders accounted for $3 \%$ and $1.5 \%$, respectively, of the exposed cohort.

Exposure to psychotropic polytherapy was reported in $72.2 \%$ of the assessed cohort. Forty-four per cent, $16.5 \%$ and $11.3 \%$ of women in the exposed group reported the use of two, three and four or more psychotropic medications, respectively. Antidepressants were most frequently used in conjunction with an SGA $(54.1 \%)$, followed by benzodiazepines (15.8\%) and anticonvulsants (14.3\%), primarily lamotrigine (table 1 ).

The mean duration of fetal exposure to an SGA was 31.2 weeks and ranged from 4 to 42 weeks. Seventy-six per cent of women reported using an SGA throughout

Table 1 List and proportion of psychotropic medications used together with an SGA

\begin{tabular}{lrl}
\hline & N & Per cent \\
\hline Selective serotonin reuptake inhibitor & 46 & 34.6 \\
Benzodiazepine & 21 & 15.8 \\
Anticonvulsant & 19 & 14.3 \\
Serotonin-norepinephrine reuptake inhibitor & 16 & 12.0 \\
Atypical antidepressant & 12 & 9.0 \\
Non-benzo hypnotic & 8 & 6.0 \\
Typical antipsychotic & 5 & 3.8 \\
Serotonin antagonist and reuptake inhibitor & 4 & 3.0 \\
Tetracyclic antidepressant & 4 & 3.0 \\
Tricyclic antidepressant & 3 & 2.3 \\
Norepinephrine reuptake inhibitor & 2 & 1.5 \\
Synthetic cannaboid & 2 & 1.5 \\
Psychostimulant & 1 & 0.8 \\
GABA analogue & 1 & 0.8 \\
Other dopamine antagonist & 1 & 0.8 \\
\hline Women taking more than two psychotropic medications may be \\
included in more than one category. \\
GABA, $\gamma$-aminobutyric acid; SGA, second generation \\
antipsychotic.
\end{tabular}


their entire pregnancy $(n=101)$, while the remaining reported exposure in at least one trimester.

\section{Perinatal outcomes of exposed and healthy comparison groups \\ Maternal characteristics}

Exposed and control women did not differ with respect to maternal age at conception. The exposed women weighed significantly more than the controls prior to conception $(p<0.001)$; however, the two groups did not differ with respect to weight gain during pregnancy. Significantly more women in the exposed group smoked cigarettes during pregnancy $(\mathrm{p}<0.001)$ and failed to use prenatal vitamins $(\mathrm{p}<0.001)$ compared with controls. Thirty-eight per cent of mothers taking SGAs did not breastfeed, which is approximately eight times greater than in controls $(p<0.001)$. Approximately two to three times as many women in the exposed group suffered from hypertension, gestational diabetes and hypothyroidism. Multilinear regression analysis was used to identify significant predictors for pre-pregnancy maternal weight gain. The only significant predictor found was group affiliation $(\mathrm{p}<0.001$; table 2$)$.

\section{Pregnancy outcomes}

The average rates of miscarriage ( $<20$ weeks) and fetal death ( $\geq 20$ weeks), $13.2 \%$ and $1.5 \%$, respectively, did not differ significantly between the two groups. The remaining analyses were conducted on live births $(\mathrm{N}=229)$. The neonates in the exposed and comparison

Table 2 Perinatal outcomes of exposed and healthy comparison groups

\begin{tabular}{|c|c|c|c|c|c|}
\hline \multirow{2}{*}{$\frac{\text { Variable }}{\text { Maternal characteristics }}$} & \multicolumn{2}{|c|}{ Exposed group } & \multicolumn{2}{|c|}{$\begin{array}{l}\text { Healthy comparison } \\
\text { group }\end{array}$} & \multirow[t]{2}{*}{ p Value } \\
\hline & $(\mathrm{N}=133)$ & & $(\mathrm{N}=133)$ & & \\
\hline & Mean & SD & Mean & $\mathrm{SD}$ & \\
\hline Age at conception (months) ${ }^{*}$ & 378.33 & 61.79 & 383.61 & 53.92 & 0.459 \\
\hline Pre-pregnancy weight $(\mathrm{kg}) \dagger$ & 74.27 & 20.86 & 64.56 & 13.01 & $<0.001$ \\
\hline \multirow[t]{2}{*}{ Maternal pregnancy weight gain $(\mathrm{kg}) \dagger$} & 15.01 & 9.33 & 14.68 & 5.57 & 0.535 \\
\hline & $\mathrm{N}$ & $\%$ & $\mathrm{~N}$ & $\%$ & \\
\hline Vitamin use & 113 & 87.6 & 131 & 99.2 & $<0.001$ \\
\hline Alcohol use $\ddagger$ & 8 & 6.0 & 12 & 9.1 & 0.343 \\
\hline Smoking $\ddagger$ & 24 & 18.0 & 0 & 0.0 & $<0.001$ \\
\hline Breastfeeding $\ddagger, \S$ & 69 & 62.2 & 100 & 95.2 & $<0.001$ \\
\hline Hypertension $\ddagger$ & 14 & 10.5 & 6 & 4.5 & 0.063 \\
\hline Gestational diabetes $\ddagger$ & 11 & 8.3 & 5 & 3.8 & 0.122 \\
\hline Hypothyroidism & 7 & 5.3 & 2 & 1.5 & 0.172 \\
\hline \multirow[t]{2}{*}{ Pregnancy outcomes for live births } & $(\mathrm{N}=113)$ & & $(\mathrm{N}=116)$ & & \\
\hline & Mean & $\mathrm{SD}$ & Mean & $\mathrm{SD}$ & \\
\hline Gestational age $\dagger$ & 39.03 & 1.86 & 39.47 & 1.49 & 0.091 \\
\hline \multirow[t]{2}{*}{ Birth weight* } & 3405.07 & 583.67 & 3365.69 & 511.09 & 0.591 \\
\hline & $\mathrm{N}$ & $\%$ & $\mathrm{~N}$ & $\%$ & \\
\hline Congenital malformations $\rrbracket$ & 7 & 6.2 & 3 & 2.6 & 0.211 \\
\hline Preterm delivery $\ddagger$ & 12 & 10.6 & 5 & 4.3 & 0.071 \\
\hline Small for gestational age ( $<10$ th centile) $\ddagger$ & 13 & 11.6 & 10 & 8.8 & 0.495 \\
\hline Large for gestational age (>90th centile) $\ddagger$ & 13 & 11.6 & 4 & 3.5 & 0.022 \\
\hline \multirow{2}{*}{ Delivery and neonatal outcomes for live births } & $(\mathrm{N}=113)$ & & $(\mathrm{N}=116)$ & & \\
\hline & $\mathrm{N}$ & $\%$ & $\mathrm{~N}$ & $\%$ & \\
\hline Method of delivery $\ddagger$ & & & & & 0.042 \\
\hline Vaginal & 78 & 69.0 & 89 & 76.7 & \\
\hline C-section repeat & 5 & 4.4 & 9 & 7.8 & \\
\hline C-section emergency & 17 & 15.0 & 15 & 12.9 & \\
\hline C-section schedules & 13 & 11.5 & 3 & 2.6 & \\
\hline Forceps/vacuum used $\ddagger$ & 10 & 8.9 & 16 & 14.0 & 0.229 \\
\hline Fetal distress $\ddagger$ & 20 & 22.5 & 15 & 14.3 & 0.140 \\
\hline Admitted to the NICU $¥$ & 23 & 25.3 & 11 & 9.5 & 0.002 \\
\hline PNA signs $\ddagger$ & 15 & 16.5 & 6 & 5.2 & 0.007 \\
\hline \multicolumn{6}{|c|}{$\begin{array}{l}\text { *Independent } t \text { test. } \\
\text { †Mann-Whitney U test. } \\
\text { †Pearson's } \chi^{2} \text { test. } \\
\text { §N (\%) of live births. } \\
\text { ๆFisher's exact test. } \\
\text { NICU, neonatal intensive care unit; PNA, poor neonatal adaptation. }\end{array}$} \\
\hline
\end{tabular}


groups did not differ in birth weight or in the proportion of those who were small for gestational age $(<10$ th centile). Exposed neonates were approximately two and a half times more likely to be premature (gestational age $<37$ weeks $)$ compared with controls $(10.6 \%$ vs $4.3 \%)$. A significantly larger proportion of exposed neonates were considered LGA ( $>90$ th centile; $\mathrm{p}=0.022)$. We found a significant correlation (at the 0.01 level, twotailed) between maternal pre-pregnancy weight and neonatal birth weight. Logistic regression analysis was performed in order to identify the significant predictors for the LGA outcome. After adjusting each model for the treatment effect, no predictors were significantly associated with the outcome. To examine predictors within the polytherapy subgroup only, unadjusted logistic regression models (due to the small sample size) were subsequently tested. Maternal pre-pregnancy weight was the only significant predictor identified $(\mathrm{p}=0.015)$.

Although not statistically significant, the rate of major malformations reported in the exposed group was approximately two and a half times higher than in the comparison group $(6.2 \%$ vs $2.6 \%)$. None of the children with major malformations were exposed to antiepileptic drugs (table 3).

\section{Delivery and neonatal outcomes}

The exposed and comparison groups differed significantly in terms of the method of delivery reported ( $\mathrm{p}=0.042)$, with more scheduled c-sections occurring in the exposed group. The two groups did not statistically differ with respect to the proportion of deliveries requiring the use of either a vacuum or forceps, or the rate of fetal distress reported during labour. Information regarding neonatal intensive care unit (NICU) admissions and neonatal signs after delivery was available for 91 exposed neonates and 116 controls. Significantly more neonates exposed to SGAs were admitted to an NICU $(p=0.002)$ and presented with poor neonatal adaptation (PNA) signs, such as central nervous system, respiratory and gastrointestinal problems, $(p=0.007)$, compared with controls. It is important to note that the vast majority of the reported differences between the exposed and comparison groups were found in patients exposed to polytherapy.

\section{Perinatal outcomes of monotherapy and polytherapy subgroups \\ Maternal characteristics}

Women receiving SGA in monotherapy were compared with those women receiving polytherapy with at least one additional psychotropic medication (table 4).

There were no differences between these two groups with respect to maternal age at conception, prepregnancy weight, prenatal vitamin use, alcohol use, smoking, maternal weight gain in pregnancy and duration of fetal exposure to SGA. Although not reaching statistical significance, more women receiving monotherapy breastfed compared with those receiving polytherapy, and women in the polytherapy group were approximately

Table 3 Descriptions of malformations reported in the assessed cohort

Congenital malformation/abnormality

\section{Exposed to}

polytherapy

Quetiapine

Fluoxetine

Clonazepam

Zoplicone

Quetiapine

Citalopram

Quetiapine

Citalopram

Quetiapine

Citalopram

Quetiapine

Atomextine

Quetiapine

Sertraline

Olanzapine

Citalopram

Healthy controls

No drug

No drug

No drug
Daily dose (mg/day)

25

80

0.5

7.5

25

60

400

20

$12.5 ; 50$

60

400

40

25

150 gradually decreased to

50

2.5

20
Timing of exposure

Throughout pregnancy

Throughout pregnancy

From week 18

Throughout pregnancy

Throughout pregnancy

Throughout pregnancy

Throughout pregnancy

From weeks 8-16

Up to week 36; from week

36

Throughout pregnancy

Throughout pregnancy

Throughout pregnancy

Up to week 7

Throughout pregnancy

Throughout pregnancy

Throughout pregnancy
Chiari malformation

Congenital heart defect

Atrial septal defect

Small bowel atresia

Hypospadias

Ureterocele with hydronephrosis of left kidney

Atrial septal defect

Macrocystic lymphatic malformation

Ventricular septal defect

Ventricular septal defect 
Table 4 Perinatal outcomes of monotherapy and polytherapy subgroups

\begin{tabular}{|c|c|c|c|c|c|}
\hline \multirow{2}{*}{$\frac{\text { Variable }}{\text { Maternal characteristics }}$} & \multicolumn{2}{|c|}{ Monotherapy } & \multicolumn{2}{|c|}{ Polytherapy } & \multirow[t]{2}{*}{ p Value } \\
\hline & $(\mathrm{N}=37)$ & & $(\mathrm{N}=96)$ & & \\
\hline & Mean & SD & Mean & SD & \\
\hline Age at conception (months) ${ }^{*}$ & 375.60 & 60.09 & 379.40 & 62.72 & 0.752 \\
\hline Pre-pregnancy weight $(\mathrm{kg}) \dagger$ & 73.94 & 18.64 & 74.41 & 21.79 & 0.750 \\
\hline \multirow[t]{2}{*}{ Maternal pregnancy weight gain $(\mathrm{kg}) \dagger$} & 14.98 & 10.84 & 15.02 & 8.79 & 0.953 \\
\hline & $\mathrm{N}$ & $\%$ & $\mathrm{~N}$ & $\%$ & \\
\hline Vitamin use & 33 & 91.7 & 80 & 86.0 & 0.554 \\
\hline Alcohol use $\ddagger$ & 2 & 5.4 & 6 & 6.3 & 1.000 \\
\hline Smoking $\ddagger$ & 5 & 13.5 & 19 & 19.8 & 0.399 \\
\hline Breastfeeding $\ddagger, \S$ & 23 & 74.2 & 46 & 57.5 & 0.104 \\
\hline Hypertension $\ddagger$ & 2 & 5.4 & 12 & 12.5 & 0.348 \\
\hline Gestational diabetes $\ddagger$ & 2 & 5.4 & 9 & 9.4 & 0.518 \\
\hline Hypothyroidism & 2 & 5.4 & 5 & 5.2 & 1.000 \\
\hline \multirow[t]{2}{*}{ Pregnancy outcomes for live births } & $(\mathrm{N}=32)$ & & $(\mathrm{N}=83)$ & & \\
\hline & Mean & $\mathrm{SD}$ & Mean & SD & \\
\hline Gestational age $†$ & 39.70 & 2.07 & 38.77 & 1.72 & 0.005 \\
\hline \multirow[t]{2}{*}{ Birth weight* } & 3426.60 & 721.61 & 3396.83 & 526.46 & 0.810 \\
\hline & $\mathrm{N}$ & $\%$ & $\mathrm{~N}$ & $\%$ & \\
\hline Congenital malformations & 0 & 0.0 & 7 & 8.4 & 0.187 \\
\hline Preterm delivery $\ddagger$ & 4 & 12.9 & 8 & 9.8 & 0.733 \\
\hline Small for gestational age ( $<10$ th centile) $\ddagger$ & 3 & 9.7 & 10 & 12.3 & 0.758 \\
\hline Large for gestational age (>90th centile) $\ddagger$ & 2 & 6.5 & 11 & 13.6 & 0.349 \\
\hline \multirow[t]{2}{*}{ Delivery and neonatal outcomes for live births } & $(\mathrm{N}=32)$ & & $(\mathrm{N}=83)$ & & \\
\hline & $\mathrm{N}$ & $\%$ & $\mathrm{~N}$ & $\%$ & \\
\hline Method of delivery $¥$ & & & & & 0.324 \\
\hline Vaginal & 23 & 74.2 & 54 & 67.1 & \\
\hline C-section repeat & 0 & 0.0 & 5 & 6.1 & \\
\hline C-section emergency & 6 & 19.4 & 11 & 13.4 & \\
\hline C-section schedules & 2 & 6.5 & 11 & 13.4 & \\
\hline Forceps/vacuum used $\ddagger$ & 0 & 0.0 & 10 & 12.3 & 0.059 \\
\hline Fetal distress $\ddagger$ & 3 & 12.0 & 17 & 26.6 & 0.139 \\
\hline Admitted to the NICU & 4 & 16.0 & 19 & 28.8 & 0.210 \\
\hline PNA signsł & 1 & 4.0 & 14 & 21.2 & 0.060 \\
\hline \multicolumn{6}{|l|}{$\begin{array}{l}\text { *Independent t test. } \\
\text { †Mann-Whitney U test. } \\
\text { †Pearson's } \chi^{2} \text { test. } \\
\text { §N (\%) of live births. } \\
\text { "IFisher's exact test. }\end{array}$} \\
\hline
\end{tabular}

twice as likely to suffer from hypertension $(12.5 \%$ vs $5.4 \%)$ and gestational diabetes $(9.4 \%$ vs $5.4 \%)$.

\section{Pregnancy outcomes}

The gestational age of neonates born to women receiving polytherapy was significantly shorter than those exposed to monotherapy ( 38.77 vs $39.70, \mathrm{p}=0.005)$. The monotherapy and polytherapy groups did not differ with respect to the rate of preterm deliveries, birth weight or proportion of small for gestational age neonates. Although not statistically significant, it is clinically relevant to report that approximately twice as many neonates exposed to polytherapy were LGA (13.6\% vs $6.5 \%)$. All seven major malformations occurring in the exposed group were associated with maternal polytherapy, which reached $8.4 \%$ for this subgroup, and no major malformations were reported in the monotherapy group.

Delivery and neonatal outcomes

All instrumental deliveries were performed in the polytherapy-exposed group $(12.3 \%$ vs $0 \%)$. When compared with the neonates exposed to monotherapy, a larger proportion of neonates in the polytherapy group were admitted to the NICU $(28.8 \%$ vs $16 \%)$ and presented with PNA signs $(21.2 \%$ vs $4 \%)$. The observed rate of PNA signs in the monotherapy group was similar to that reported in the healthy comparison group (4\% vs $5.2 \%)$. Ninety-three per cent of the exposed neonates that presented with PNA symptoms were born to mothers receiving psychotropic polytherapy, and $53 \%$ of 
these neonates were prenatally exposed to a selective serotonin reuptake inhibitor (SSRI). Statistical significance was not reached for these outcomes.

\section{DISCUSSION}

At present, this is one of the largest studies to investigate pregnancy outcomes following maternal use of SGA monotherapy and polytherapy with other psychotropic medications. Our data support an increasing trend in the use of SGAs to treat a variety of psychiatric disorders, with bipolar disorder and depression being reported as the two leading indications and quetiapine being the one most commonly used. We found that $76 \% \quad(n=101)$ of women were exposed to SGAs throughout pregnancy and $72 \%$ of the assessed cohort received psychotropic drugs in polytherapy, which is in agreement with previous reports. ${ }^{161921} 22$ Frye $e t a t^{23}$ stated that the proportion of polytherapy treatment increased from $3.3 \%$ in $1974-1979$ to $44 \%$ in $1990-1995 .{ }^{24}$ Recent studies indicate that this trend towards polytherapy continues to rise, reaching up to $80 \% .^{22}$ Interestingly, Centorrino and colleagues maintained that there are no apparent advantages in opting for treatments with multiple antipsychotics. Fifty-four per cent of women exposed to polytherapy in our study were treated with both an SGA and an antidepressant. These findings are consistent with recent reports of increased polytherapy involving antipsychotics and antidepressants in the USA, ${ }^{20}$ as well as in other cohorts of pregnant women. ${ }^{14161921}$

We found that women in the exposed and comparison groups differed significantly with respect to a number of characteristics and behaviours that are known to be associated with increased risks for adverse pregnancy outcomes. Exposed women weighed significantly more prior to becoming pregnant, although body mass index could not be calculated due to the lack of available data on maternal height. This finding is consistent with reports of significant weight gain induced by SGAs. ${ }^{25-28}$ There were no differences in maternal weight gain during pregnancy, and as such, more consideration should be given to the management of preconceptional weight. In addition, significantly higher proportions of exposed women smoked more cigarettes during pregnancy and were less likely to breastfeed and take prenatal vitamins. The increased proportion of preterm deliveries observed in the exposed group may be influenced by maternal pre-pregnancy weight, as was reported in a recent paper by Khatibi et al..$^{29}$ Moreover, in agreement with a recent population-based cohort study that reported an increased incidence of gestational diabetes in mothers taking SGAs during pregnancy, ${ }^{28}$ the exposed women in our cohort experienced more associated comorbidities, including hypertension, gestational diabetes and hypothyroidism. Although these latter findings were not statistically significant, they may be of clinical relevance. However, the exposed group did have a significantly increased rate of LGA neonates. It is of interest to note that, in spite of the large proportion of exposed women who exhibited characteristics commonly associated with risk for small for gestational age neonates (eg, smoking, hypertension), an increased rate of LGA neonates was observed. Such results may be considered in context with exposure to psychotropic drugs. Moreover, the exposed neonates were admitted more often to the NICU, and a greater proportion presented with PNA signs. Six per cent of exposed infants presented with major malformations compared with $2.6 \%$ in the comparison group. Although $14.3 \%$ of exposed mothers were also taking an antiepileptic drug, primarily lamotrigine, the reported malformations were not found among those infants exposed to anticonvulsants.

The present report is one of the first to compare the pregnancy outcomes of women receiving SGA monotherapy with those receiving SGAs in polytherapy. Although women from both the monotherapy and polytherapy subgroups did not differ in their age at conception, prepregnancy weight, pregnancy weight gain and neonatal birth weight, the women exposed to polytherapy took fewer vitamins, smoked more cigarettes, experienced a significantly shorter gestation, tended to breastfeed less and delivered twice as many LGA neonates. Fetal distress and admission to the NICU were reported approximately twice as often in the polytherapy subgroup. Twice as many women exposed to polytherapy experienced hypertension and gestational diabetes comorbidities. All 12\% of the instrumental deliveries were observed in the polytherapy subgroup. Neonates exposed to polytherapy were five times more likely to present with PNA signs compared with those exposed to monotherapy. All the malformations reported were observed in the neonates exposed to polytherapy. Although some of these differences did not reach statistical significance (table 4), it appears that polytherapy with SGAs and other psychotropic medications may be associated with a higher risk for adverse pregnancy outcomes, compared with SGA monotherapy. The reported results should be considered with caution, given the small sample size of our cohort and that previously published case reports have observed healthy outcomes in neonates/infants prenatally exposed to SGA polytherapy. ${ }^{30-34}$

Maternal pre-pregnancy characteristics and behavioural risk factors may be considered when interpreting the findings of the present study. For example, maternal pre-pregnancy weight, specifically being overweight or obese, has been shown to be independently associated with an elevated risk for LGA births. ${ }^{35-38}$ Although this study is the first to corroborate Newham et $a l^{19}{ }^{19}$ report of increased risks of LGA deliveries in women exposed to SGAs, the study design was not able to adequately separate the effect of pre-pregnancy weight on this outcome. Thus, it is unclear whether exposure to SGAs may cause fetal-weight gain directly or indirectly by inducing maternal weight gain prior to conception. Nevertheless, the findings are in contrast to a previously 
published study which found that in utero exposure to SGAs was associated with a significantly elevated risk of small for gestational age births. ${ }^{16}$ However, it is possible that this difference may be due to the lack of control of potential confounders, such as maternal indication for smoking or substance use during pregnancy. Twenty-four per cent of women in this earlier study reportedly suffered from schizophrenia, a severe psychiatric disorder that is independently associated with an increased risk for small for gestational age and low birth weight births. ${ }^{17}$ In contrast, only $3 \%$ of women in the present study reported a diagnosis of schizophrenia. Similarly, $38 \%$ of exposed women in this earlier study, in contrast to $18 \%$ in the current study, reported smoking during pregnancy-a risk factor that has also been associated with elevated risks of small for gestational age ${ }^{39-41}$ and intrauterine growth-restricted ${ }^{42}$ neonates. Further studies controlling for known predictors of LGA are necessary to confirm the findings, and an exploration of the potential biological mechanisms underpinning this phenomenon is warranted.

To our knowledge, the present study is the first to show a significant association between maternal SGA use in pregnancy and an elevated risk of NICU admission. The $25 \%$ prevalence rate of NICU admissions found in the exposed group of the present study is the same as that reported in a recent retrospective chart review, although only 16 women were assessed. ${ }^{43}$

Our results also suggest that polytherapy in pregnancy may be associated with shorter gestation and increased rates of LGA neonates, instrumental deliveries, fetal distress during delivery, NICU admissions and neonates presenting with PNA symptoms. Such findings may have important clinical relevance, as the rates of many adverse delivery/neonatal outcomes were at least twice as high in the polytherapy group. Importantly, among the exposed group, all seven major malformations occurred among the 96 women receiving polytherapy; however, no specific pattern of malformation was found. The association between polytherapy exposure and higher rates of malformations should be considered in the context of the small sample size, severity of the baseline disorder that required polytherapy, associated comorbidities, as well as any other unknown environmental factors that were not assessed in this study. Considering that exposure to SGA monotherapy was not associated with birth defects in our cohort and that the majority of pregnant women with psychiatric disorders are on psychotropic drug polytherapy, research on the reproductive safety of these medications should reflect this reality. Moreover, further research is required in order to separate the effects of polytherapy from the baseline mental illness, as well as the genetic and environmental confounders on pregnancy outcome, particularly as those receiving psychotropic polytherapy may suffer from more severe and persistent psychiatric disorders.

The higher prevalence of PNA signs found in the exposed group, compared with the controls, may be driven by concurrent SSRI exposure, as 12 of the 15 neonates that presented with PNA symptoms were also exposed to an SSRI. Although the measurement scales specific for the PNA syndrome are not available, numerous studies have found that use of SSRI late in pregnancy is associated with an increased risk of infants developing this clinical presentation, ${ }^{44}{ }^{45}$ which is also labelled as Prenatal Antidepressant Exposure Syndrome. ${ }^{46}$ The three remaining neonates in our cohort who displayed PNA symptoms were prenatally exposed to an SGA but not to an SSRI. Similar to the present study, Mendhekar et $a l^{47}$ in 2006 described a neonate who presented with unexplained fetal distress following prenatal exposure to aripiprazole. Such findings have been echoed by the US Food and Drug Administration in recently issued warnings suggesting that, similar to SSRIs, antipsychotics may cause PNA syndrome. ${ }^{48}$ In the present study, $82.6 \%$ of neonates admitted to the NICU were exposed to polytherapy, mostly with SSRIs. Given the predominance of polytherapy involving these two classes of medications in the treatment of various psychiatric disorders in pregnant women, designing future cohort studies aimed at investigating the relative and combined effects of prenatal exposure to SGAs and antidepressants is of considerable importance.

There are a number of limitations inherent in the design of the present study that should be acknowledged. Given the small sample size, unadjusted models were used in most of the analyses. Therefore, we were unable to fully assess the influence of confounding factors in SGA monotherapy. Such results should be further investigated in future research. Another limitation is the inability to address confounding by indication and separate the relative effects of maternal mental illness from psychotropic pharmacotherapy on pregnancy outcome. Moreover, a more detailed investigation into maternal mental health, which includes measures of the frequency and severity of specific psychiatric symptoms during pregnancy, would allow for a more thorough investigation into these critical confounding variables. Furthermore, evaluating the social characteristics of the assessed cohort would enable us to identify potential confounding variables that may affect pregnancy outcomes, such as socioeconomic status and lifestyle differences. The women who contacted Motherisk tended to be middle to upper-middle class, which represents the majority of the population, and may be higher functioning than those who did not, as they independently sought access to counselling in order to help educate themselves on the potential teratogenic risks associated with their medications. ${ }^{16}$ A smaller proportion of callers came from the lower socioeconomic and/ or immigrant population; however, this may not affect the generalisability of the results, as their outcomes may be confounded by other factors unrelated to the drug effect. Although the women were recruited prospectively, there are potential limitations in the retrospectivity of a database. 
In conclusion, it appears that psychotropic polytherapy in pregnant women with mental health disorders is presently a common practice. However, such management may lead to an increased risk of adverse pregnancy outcomes. Considering that reports that polytherapy do not commonly alter the course and outcome of the baseline disorder, ${ }^{22} 24$ indications for polytherapy in pregnant women should be seriously considered and made on an individual basis following a thorough risk/benefit analysis. The reproductive safety of antipsychotic medications should be studied using a prospective design and a powerful sample size and in the reality of polytherapy, associated comorbidities and environmental and genetic confounders.

\section{Author affiliations}

${ }^{1}$ Motherisk Program, Division of Clinical Pharmacology and Toxicology,

The Hospital for Sick Children, Toronto, Ontario, Canada

${ }^{2}$ York University, Toronto, Ontario, Canada

${ }^{3}$ University of Toronto, Toronto, Ontario, Canada

Contributors IN, GK and PYB designed the study. AS and MT carried out the patient recruitment and interviews, created the database, and wrote the initial draft of the paper. IN and MT performed the statistical analysis, and IN supervised the data collection, handling and analysis. IN, GK and PYB contributed to the interpretation, as well as to manuscript preparation and critical revision. As the guarantor, IN had full access to all the data (including statistical reports and tables) and the database in the study and can take responsibility for the integrity of the data and the accuracy of the data analysis. Each author participated in the preparation and review of this manuscript and gave the final approval for the submission of this manuscript.

Funding Motherisk Funds.

Competing interests None.

Ethics approval The Research Ethics Board at The Hospital for Sick Children granted approval for this study (reference number: 1000013674) on 26 March 2009.

Provenance and peer review Not commissioned; externally peer reviewed.

Data sharing statement Motherisk owns this prospectively collected database from where the information was used

\section{REFERENCES}

1. Vesga-López O, Blanco $\mathrm{C}$, Keyes $\mathrm{K}$, et al. Psychiatric disorders in pregnant and postpartum women in the United States. Arch Gen Psychiatry 2008;65:805-15.

2. Andersson L, Sundstrom-Poromaa I, Bixo M, et al. Point prevalence of psychiatric disorders during the second trimester of pregnancy: a population-based study. Am J Obstet Gynecol 2003;189:148-54.

3. Sundström IM, Bixo M, Björn I, et al. Prevalence of psychiatric disorders in gynecologic outpatients. Am J Obstet Gynecol 2001;184:8-13.

4. Oyebode F, Rastogi A, Giles B, et al. Psychotropics in pregnancy: safety and other considerations. Pharmacol Ther 2012;135:71-7.

5. Nulman I, Citron S, Todorow M, et al. Neurodevelopment of children exposed to antidepressant and antipsychotic medications during pregnancy. In: Preece PM, Riley EP, eds. Alcohol, drugs and medication in pregnancy: the long-term outcome for the child. London, England: Mac Keith Press, 2011:56-84.

6. Nilsson E, Lichtenstein $\mathrm{P}, \mathrm{Cnattingius} \mathrm{S}$, et al. Women with schizophrenia: pregnancy outcome and infant death among their offspring. Schizophr Res 2002;58:221-9.

7. Davalos D, Yadon C, Tregellas H. Untreated prenatal maternal depression and potential risks to offspring: a review. Arch Womens Ment Health 2012;15:1-14.

8. Lee HC, Lin HC. Maternal bipolar disorder increased low birthweight and preterm births: a nationwide population-based study. J Affect Disord 2012;121:100-5.
9. Gentile S. Antipsychotic therapy during early and late pregnancy. A systematic review. Schizophr Bull 2010;36:518-44.

10. Alexander GC, Gallagher SA, Mascola A, et al. Increasing off-label use of antipsychotic medication in the United States, 1995-2008. Pharmacoepidem Dr S 2011;20:177-84

11. Ahearn EP, Juergens $T$, Cordes $T$, et al. A review of atypical antipsychotic medications for posttraumatic stress disorder. Int Clin Psychopharmacol 2011;26:193-200.

12. Barbui C, Danese A, Guaiana G, et al. Prescribing second-generation antipsychotics and the evolving standard of care in Italy. Pharmacopsychiatry 2002;35:239-43.

13. Rowe DL. Off-label prescription of Quetiapine in psychiatric disorders. Expert Rev Neurother 2007;7:841-52.

14. Newport DJ, Calamaras MR, DeVane CL, et al. Atypical antipsychotic administration during late pregnancy: placental passage and obstetrical outcomes. Am J Psychiatry 2007;164:1214-20.

15. Johnson KC, LaPrairie JL, Brennan PA, et al. Prenatal antipsychotic exposure and neuromotor performance during infancy. Arch Gen Psychiatry 2012;69:787-94

16. McKenna K, Koren G, Tetelbaum M, et al. Pregnancy outcome of women using atypical antipsychotic drugs: a prospective comparative study. J Clin Psychiatry 2005;66:444-9.

17. Lin H, Chen I, Chen $\mathrm{Y}$, et al. Maternal schizophrenia and pregnancy outcome: does the use of antipsychotics make a difference? Schizophr Res 2010;116:55-60.

18. Reis M, Källén B. Maternal use of antipsychotics in early pregnancy and delivery outcome. J Clin Psychopharmacol 2008;28:279-88.

19. Newham JJ, Thomas SH, MacRitchie K, et al. Birth weight of infants after maternal exposure to typical and atypical antipsychotics: prospective comparison study. Br J Psychiatry 2008;192:333-7.

20. Mojtabai R, Olfson M. Psychotropic medication polypharmacy in office-based psychiatry in the United States. Arch Gen Psychiatry 2010;67:26-36.

21. Hironaka M, Kotani T, Sumigama S, et al. Maternal mental disorders and pregnancy outcomes: a clinical study in a Japanese population. J Obstet Gynaecol Res 2011;37:1283-9.

22. Centorrino F, Cincotta SL, Talamo A, et al. Hospital use of antipsychotic drugs: polytherapy. Compr Psychiatry 2008;49:65-9.

23. Frye MA, Ketter TA, Leverich GS, et al. Increasing use of polypharmacotherapy for refractory mood disorders: 22 years of study. J Clin Psychiatry 2000;61:9-15.

24. Centorrino F, Goren JL, Hennen J, et al. Multiple versus single antipsychotic agents for hospitalized psychiatric patients: case-control study of risks versus benefits. Am J Psychiatry 2004;161:700-6.

25. Taylor DM, McAskill R. Atypical antipsychotics and weight gain-a systematic review. Acta Psychiatr Scand 2000;101:416-32.

26. Gentile S. Long-term treatment with atypical antipsychotics and the risk of weight gain: a literature analysis. Drug Saf 2006;29:303-19.

27. Newcomer JW. Second-generation (atypical) antipsychotics and metabolic effects: a comprehensive literature review. CNS Drugs 2005;19(Suppl 1):1-93.

28. Bodén R, Lundgren M, Brandt $L$, et al. Antipsychotics during pregnancy: relation to fetal and maternal metabolic effects. Arch Gen Psychiatry 2012;69:715-21.

29. Khatibi A, Brantsaeter AL, Sengpiel V, et al. Prepregnancy maternal body mass index and preterm delivery. Am J Obstet Gynecol 2012;207:212.e1-7

30. Yaris F, Yaris E, Kadioglu M, et al. Use of polypharmacotherapy in pregnancy: a prospective outcome in a case. Prog Neuro-Psychopharmacol Biol Psychiatry 2004;28:603-5.

31. Gentile S. Quetiapine-fluvoxamine combination during pregnancy and while breastfeeding. Arch Womens Ment Health 2006;9:158-9.

32. Çabuk D, Sayin A, Derinöz O, et al. Quetiapine use for the treatment of manic episode during pregnancy. Arch Womens Ment Health 2007;10:235-6.

33. Vemuri MP, Rasgon NL. A case of olanzapine-induced gestational diabetes mellitus in the absence of weight gain. J Clin Psychiatry 2007;68:1989.

34. Aichhorn $\mathrm{W}$, Yazdi $\mathrm{K}$, Kralovec $\mathrm{K}$, et al. Olanzapine plasma concentration in a newborn. J Psychopharmacol 2008;22:923-4.

35. Schaefer-Graf UM, Heuer R, Kilavuz O, et al. Maternal obesity not maternal glucose values correlates best with high rates of fetal macrosomia in pregnancies complicated by gestational diabetes. J Perinat Med 2002;30:313-21.

36. Ehrenberg HM, Mercer BM, Catalano PM. The influence of obesity and diabetes on the prevalence of macrosomia. Am J Obstet Gynecol 2004;191:964-8.

37. Ricart W, López J, Mozas J, et al. Spanish group for the study of the impact of carpenter and coustan GDM thresholds: body mass index 
has a greater impact on pregnancy outcomes than gestational hyperglycaemia. Diabetologia 2005;48:1736-42.

38. Nielsen GL, Dethlefsen C, Møllert M, et al. Maternal glycated hemoglobin, pre-gestational weight, pregnancy weight and risk of large-for-gestational-age neonates: a Danish cohort study of 209 singleton Type 1 diabetic pregnancies. Diabet Med 2007;24:384-7.

39. Thompson JM, Clark PM, Robinson E, et al. Risk factors for small-for-gestational-age neonates: the Auckland Birthweight Collaborative Study. J Paediatr Child Health 2001;37:369-75.

40. Mitchell EA, Thompson JM, Robinson E, et al. Smoking, nicotine and tar and risk of small for gestational age neonates. Acta Paediatr 2002:91:323-8.

41. Fantuzzi G, Vaccaro V, Aggazzotti G, et al. Exposure to active and passive smoking during pregnancy and severe small for gestational age at term. J Matern-Fetal Neo M 2008;21:643-7.

42. Horta BL, Victora CG, Menezes AM, et al. Low birthweight, preterm births and intrauterine growth retardation in relation to maternal smoking. Paediatr Perinat Epidemiol 1997;11:140-51.
43. Wichman C. Atypical antipsychotic use in pregnancy: a retrospective review. Arch Womens Ment Health 2009;12:53.

44. Moses-Kolko EL, Bogen D, Perel J, et al. Neonatal signs after late in utero exposure to serotonin reuptake inhibitors: literature review and implications for clinical applications. JAMA 2005;293:2372-83.

45. Gentile S. Serotonin reuptake inhibitor-induced perinatal complications. Pediatric Drugs 2007;9:97-106

46. Gentile S. On categorizing gestational, birth, and neonatal complications following late pregnancy exposure to antidepressants: the prenatal antidepressant exposure syndrome. CNS Spectr 2010;5:167-85.

47. Mendhekar DN, Sunder KR, Andrade C. Aripiprazole use in a pregnant schizoaffective woman. Bipolar Disord 2006;8:299-300.

48. U.S. Food and Drug Administration. FDA Drug Safety

Communication: Antipsychotic drug labels updated on use during pregnancy and risk of abnormal muscle movements and withdrawal symptoms in newborns. 2011. http://www.fda.gov/NewsEvents/ Newsroom/PressAnnouncements/2008/ucm116912.htm 